\section{Biodegradable multi-drug dispenser}

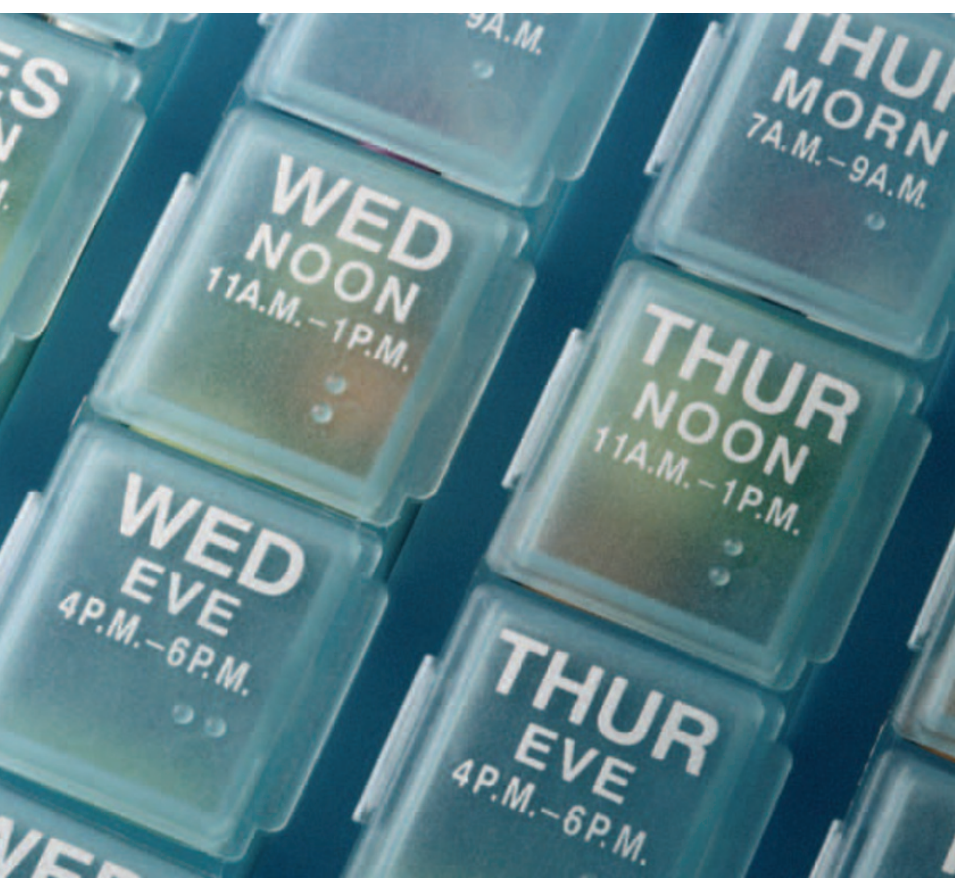

Have you ever forgotten to take your daily pills at the right time? Or missed one altogether? Although important, forgetfulness is not the only problem with drug delivery. Traditionally, most drugs are delivered by oral or intravenous means, which can lead to high concentrations of drugs in the bloodstream with concomitant toxic side effects, and yet only a small percentage of the drug actually reaches the target area. An ideal drug delivery system would maintain optimum therapeutic concentrations of the drug in the target tissue, with minimum fluctuation and allow reproducible release of the drug for long periods of time. In a study published in the November issue of Nature Materials, Langer and colleagues from MIT report a biodegradable drug delivery system with the potential to release pulses of different drugs at various intervals after implantation by using materials of different molecular masses for the membranes covering the drug-containing reservoirs.

The microchip device, made from a degradable polymer, was designed to achieve multi-pulse drug release over periods of several months, without requiring a stimulus to trigger the drug release. Reservoirs machined through a polymer disc were blocked on one side with a layer of degradable polyester tape, loaded with the drug to be released and then sealed with degradable polymeric membranes. Drugs could be released at defined times on the basis of the characteristics of the reservoir membranes. The material used, the molecular mass and the thickness all contribute to the degradation rate of the membrane, and therefore the rate of drug release. The devices were about $11.9 \mathrm{~mm}$ in diameter and about $500 \mu \mathrm{m}$ thick.

Proof-of-principle studies were conducted in vitro with microchips made from poly(L-lactic acid) (PLLA) that used poly(lactic-co-glycolic acid) (PLGA) reservoir membranes of various molecular masses to control the release of test chemicals dextran, heparin and human growth hormone. A 50/50 ratio of lactic acid/glycolic acid was chosen for the membrane as this provides a degradation time of a few weeks to months. In addition, a range of PLGA molecular masses $4,400,11,000,28,000$ and $64,000-$ were chosen. The results showed four

\section{ANTIPYRETIC DRUGS}

\section{Heat relief}

Inflammatory molecules called prostaglandins (PGs) facilitate induction of the febrile response to infection. Feveralleviating drugs, such as ibuprofen, are used by millions of people every day, and act by inhibiting cyclooxygenase (COX), which catalyses the first step in the formation of PGs from arachidonic acid. But the use of COX inhibitors is associated with severe side effects, such as gastric intolerance and depression of blood clotting, which are probably related to their non-specific effects on the synthesis of several PGs. Targeting PGs that are specifically involved in eliciting fever might permit the development of antipyretic drugs with an improved safety profile. A study published recently in Nature Neuroscience brings us one step closer to realizing this goal.

A team led by Anders Blomqvist investigated the role of microsomal prostaglandin E synthase-1 (mPGES-1) - which catalyses the second step in $\mathrm{PGE}_{2}$ production - in the febrile response, by knocking out its expression in mice. Mutant mice were indistinguishable from their wildtype littermates under normal physiological conditions. However, differences emerged following immune challenge with bacterial cell-wall lipopolysaccharide (LPS). Shortly after injection of LPS, the core body temperature of wild-type mice increased significantly and remained elevated for about six hours. By contrast, the temperature of immune-challenged mPGES-1-deficient mice did not differ from that of saline-injected controls. Direct injection of $\mathrm{PGE}_{2}$ into the brains of mutants elicited a robust febrile response, confirming that these mPGES-1-deficient mice retained the capacity to respond to the product of mPGES- 1 activity.

Levels of $\mathrm{PGE}_{2}$ in cerebrospinal fluid after administration of LPS mimicked the temperature pattern, increasing in wild-type subjects and remaining static in their mutant counterparts. Incubation of brain homogenates from immune-challenged mice with $\mathrm{PGH}_{2}$, the substrate of mPGES-1, showed that these responses were correlated with enzymatic activity, or lack thereof, in the microsomal fraction. Reverse transcriptionpolymerase chain reaction was used to confirm that the physiological effects of LPS injection were due to differential expression of mPGES-1 in brain homogenates of wildtype and mutant mice.

Interestingly, expression of another PG-synthesizing enzyme - mPGES-2 was not upregulated by immune challenge in wild-type mice, indicating that mPGES-1 is specifically involved in facilitating fever. Similarly, the response of COX-2 to LPS injection was not affected by the mPGES-1 mutation, being upregulated in both mutant and wild-type mice. These results show that, unlike present-generation COX inhibitors, compounds that target mPGES- 1 should specifically inhibit the synthesis of feverinducing $\mathrm{PGE}_{2}$, without affecting the production of other PGs. Side effects of antipyretic drugs might thereby become a thing of the past.

Suzanne Farley

(i) References and links

ORIGINAL RESEARCH PAPER Engblom, D. et al.

Microsomal prostaglandin E synthase-1 is the central switch during immune-induced pyresis. Nature Neurosci. 6 1137-1138 (2003) 
